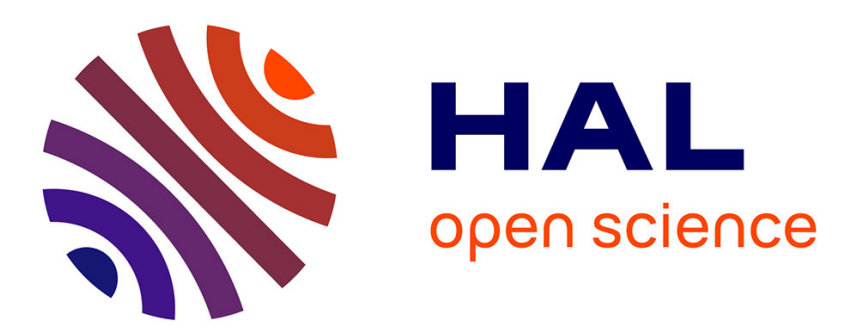

\title{
Analyse des photocourants dans les structures Al-Al2o 3-Al préparées par bombardement électronique
}

\author{
R. Jerisian, J. Dugas, Y. Mentalecheta, J. Oualid
}

\section{To cite this version:}

R. Jerisian, J. Dugas, Y. Mentalecheta, J. Oualid. Analyse des photocourants dans les structures Al-Al2o 3-Al préparées par bombardement électronique. Revue de Physique Appliquée, 1977, 12 (5), pp.773-780. 10.1051/rphysap:01977001205077300 . jpa-00244240

\section{HAL Id: jpa-00244240 https://hal.science/jpa-00244240}

Submitted on 1 Jan 1977

HAL is a multi-disciplinary open access archive for the deposit and dissemination of scientific research documents, whether they are published or not. The documents may come from teaching and research institutions in France or abroad, or from public or private research centers.
L'archive ouverte pluridisciplinaire HAL, est destinée au dépôt et à la diffusion de documents scientifiques de niveau recherche, publiés ou non, émanant des établissements d'enseignement et de recherche français ou étrangers, des laboratoires publics ou privés. 


\title{
ANALYSE DES PHOTOCOURANTS DANS LES STRUCTURES Al-Al $\mathrm{O}_{3}$-Al PRÉPARÉES PAR BOMBARDEMENT ÉLECTRONIQUE
}

\author{
R. JERISIAN, J. DUGAS et Y. MENTALECHETA \\ Département d'Etat Solide, Centre des Sciences et de la Technologie Nucléaires, \\ Boulevard Frantz-Fanon, Alger, Algérie

\section{J. OUALID} \\ Laboratoire de Photoélectricité, Faculté des Sciences et Technịques de St-Jérôme, \\ Université d'Aix-Marseille III, France
}

\begin{abstract}
Résumé. - Ce travail a consisté à étudier et analyser les photocourants dans les structures $\mathrm{Al}-\mathrm{Al}_{2} \mathrm{O}_{3}-\mathrm{Al}$ dans lesquelles la couche isolante est obtenue par bombardement électronique d'une cible d'alumine. Les analyses par rétrodiffusion nucléaire et par diffraction de rayons X ont montré que les couches d'alumine étaient stœchiométriques et amorphes.

La hauteur de barrière de l'interface $\mathrm{Al}-\mathrm{Al}_{2} \mathrm{O}_{3}$ déterminée par photoémission interne se situe à $3,2 \mathrm{eV} \pm 0,2 \mathrm{eV}$. Les valeurs plus faibles rencontrées dans la bibliographie sont attribuées soit à l'oxydation de l'électrode d'aluminium, soit à l'influence d'un courant éventuel de photoconduction qui peut se superposer au courant de photoémission au voisinage du seuil. Cette dernière hypothèse est étayée par l'étude de la variation spectrale de l'absorption des couches d'alumine.

L'action des pièges permet de rendre compte de la variation des photocourants $J_{\mathrm{ph}}$ en fonction du temps et de la fréquence de modulation, des courbures des diagrammes de Fowler des structures les plus épaisses, et de celles des caractéristiques $J_{\mathrm{ph}}=f(V)$.
\end{abstract}

\begin{abstract}
We have studied the photocurrents in $\mathrm{Al}_{-} \mathrm{Al}_{2} \mathrm{O}_{3}$ structures in which the insulating layer is obtained by electron beam evaporation of an $\mathrm{Al}_{2} \mathrm{O}_{3}$ target. The analysis by nuclear back scattering and $\mathrm{X}$-diffraction have shown that the $\mathrm{Al}_{2} \mathrm{O}_{3}$ layers were stoechiometric and amorphous.

The $\mathrm{Al}-\mathrm{Al}_{2} \mathrm{O}_{3}$ interfacial barrier height determined by internal photoemission is found to be $3.2 \mathrm{eV} \pm 0.2 \mathrm{eV}$. Lower values found in the litterature may be attributed either to aluminium oxydation or to influence of a possible additional photoconduction current near the photoemission edge. This last assumption is supported by the spectral variation of the aluminium oxyde layers optical absorption.

The trapping effects account for the variation of the photocurrent $J_{\mathrm{ph}}$ versus time and chopping frequency, for the non linearity of Fowler plots obtained with thicker structures and of $J_{\mathrm{ph}}=f(V)$ characteristics.
\end{abstract}

1. Introduction. - Parmi les méthodes de détermination des hauteurs de barrière de potentiel caractéristiques des interfaces des dispositifs M. I. S., M. I. M. ou Schottky, celle basée sur l'effet de photoémission interne est considérée comme la plus simple, la plus directe et la plus précise $[1,2,3]$. Il suffit, en principe, de déterminer le seuil du photocourant $J_{\mathrm{ph}}$ ou du rendement de photoémission

$$
R=\frac{J_{\mathrm{ph}} \cdot h v}{I_{0} \cdot T}\left({ }^{1}\right)
$$

lorsque la structure est éclairée par un rayonnement monochromatique de longueur d'onde variable.

(1) $I_{0}$ : intensité du rayonnement incident; $T$ : facteur de transmission de l'électrode supérieure.
Le seuil est obtenu par divers procédés qui consistent à :

- Déterminer dans un diagramme de Fowler $(\sqrt{R}=f(h v))$ l'intersection de la droite qui passe par le plus grand nombre de points expérimentaux avec l'axe des énergies.

- Comparer les graphes théoriques et expérimentaux représentant la variation de $\log R$ en fonction du paramètre sans dimension $x=h v / k T$ [4].

- Utiliser une méthode numérique [5].

- Exploiter les caractéristiques $J_{\mathrm{ph}}$ en fonction de la tension de polarisation pour diverses énergies $h v$ des photons [6].

Ces différents procédés conduisent à des déterminations de seuils sensiblement identiques lorsque les structures ne présentent aucune difficulté d'interprétation. 


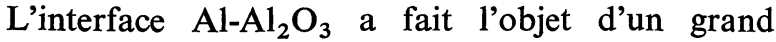
nombre d'études par la méthode de photoémission interne comme l'indique le tableau I où sont consignés les hauteurs de barrière de potentiel relatives à cette interface, obtenues par divers auteurs. La grande dispersion des résultats $\left(0,9 \mathrm{eV}<\Phi_{\mathrm{BE}}<2 \mathrm{eV}\right.$ et $1,8 \mathrm{eV}<\Phi_{\mathrm{TE}}<3,5 \mathrm{eV}$ ) est souvent attribuée au mode de préparation de l'alumine $[19,20]$. Aussi ont-ils été classés suivant ce critère.

L'analyse du tableau I montre que :

- Les structures $\mathrm{Al}-\mathrm{Al}_{2} \mathrm{O}_{3}-\mathrm{Al}$ présentent une barrière de potentiel dissymétrique $\left(\Phi_{\mathrm{TE}}>\Phi_{\mathrm{BE}}\right)$.

- La dispersion persiste quel que soit le mode

\section{TABLEAU I}

Hauteurs de barrière de l'interface $\mathrm{Al}_{2} \mathrm{O}_{3}-\mathrm{Al}$

\begin{tabular}{|c|c|c|c|c|c|c|c|}
\hline Réf. & $\underline{\varepsilon_{\mathrm{r}}}$ & $e_{\mathrm{TE}}(\AA)$ & $s(\AA)$ & $\begin{array}{l}\text { Technique } \\
\text { de mesure } \\
\end{array}$ & $\begin{array}{l}\text { Domaine } \\
\text { énergétique } \\
\text { (eV) }\end{array}$ & $\begin{array}{l}\Phi_{\mathrm{TE}} \\
(\mathrm{eV}) \\
-\end{array}$ & $\begin{array}{l}\Phi_{\mathrm{BE}} \\
(\mathrm{eV}) \\
-\end{array}$ \\
\hline \multicolumn{8}{|c|}{ Oxydation thermique } \\
\hline $\begin{array}{l}{[7]} \\
{[8]}\end{array}$ & 8 & $\begin{array}{c}100 \\
\text { transp. }\end{array}$ & $\begin{array}{c}80-120 \\
50-60\end{array}$ & $\begin{array}{l}\text { P. A. } \\
\text { D. S. à } 9 \mathrm{~Hz}\end{array}$ & $\begin{array}{c}1 \text { à } 5 \\
1,3 \text { à } 3,5\end{array}$ & $\begin{array}{l}2,9 \\
2,45\end{array}$ & $\begin{array}{l}1 \\
0,9\end{array}$ \\
\hline
\end{tabular}

\section{Electrolyse}

\begin{tabular}{|c|c|c|c|c|c|c|}
\hline [9] & 300 & 60 & D. S. à $80 \mathrm{~Hz}$ & 1,4 à 3,5 & 3,07 & 1,62 \\
\hline $\begin{array}{l}2,3 \\
\text { opt. }\end{array}$ & $\begin{array}{l}200 \\
(\mathrm{Au})\end{array}$ & 60 & D. S. à $80 \mathrm{~Hz}$ & 1,5 à 6 & $\begin{array}{l}4 \\
(\mathrm{Au})\end{array}$ & 1,8 \\
\hline \multirow{4}{*}{ [11] } & 200 & 52 & & & $2,1 \pm 0,1$ & - \\
\hline & & 78 & $\begin{array}{c}\text { D. S. de } \\
70 \text { à } 100 \mathrm{~Hz}\end{array}$ & 1,2 à 4 & $2,2 \pm 0,1$ & - \\
\hline & & $\begin{array}{l}104 \\
130\end{array}$ & & & $\begin{array}{l}2,3 \pm 0,1 \\
2,4 \pm 0,1\end{array}$ & - \\
\hline & $\begin{array}{c}100 \\
(\mathrm{Au})\end{array}$ & 170 & - & 1,2 à 2,5 & $\begin{array}{l}4 \\
(\mathrm{Au})\end{array}$ & $1,5 \pm 0,1$ \\
\hline 8,8 & $40-80$ & $400-2000$ & - & 1,8 à 5 & 3,5 & - \\
\hline
\end{tabular}

Anodisation dans un plasma d'oxygène

$\begin{array}{lllccccc}{[14]} & 8 & - & 20-40 & - & 1 \text { à } 3 & 1,92 & 1,49 \\ {[15]} & - & \text { transp. } & 40 & - & 1 \text { à } 4,2 & 1,96 & - \\ {[13]} & 8,8 & 40-80 & 400-2000 & - & 1,8 \text { à } 5 & 3,5 \pm 0,2 & - \\ {[16]} & 8,4 & \text { transp. } & 15-35 & \text { P. A. } & 1,5 \text { à } 3,5 & 2,2 & 1,75 \\ {[6]} & 8,4 & e_{\mathrm{BE}} 170 & 40 & \text { D. S. à } 9 \mathrm{~Hz} & - & 1,8 & - \\ {[17]} & 8 & 50 & 59 & \text { D. S. à } 22,6 \mathrm{~Hz} & 1,5 \text { à } 3,5 & 2,16 & 1,78 \\ & & & 49 & & & 2,05 & 1,84 \\ {[18]} & 8 & 20 \text { à } 300 & 20 \text { à } 200 & \text { D. S. à } 300 \mathrm{~Hz} & 1,5 \text { à } 3,5 & 2,4 & 1,8 \\ {[19]} & - & \text { eau } & 200-350 & \text { P. A. } & 2 \text { à } 5,5 & - & 2 \text { ou } 3,1\end{array}$

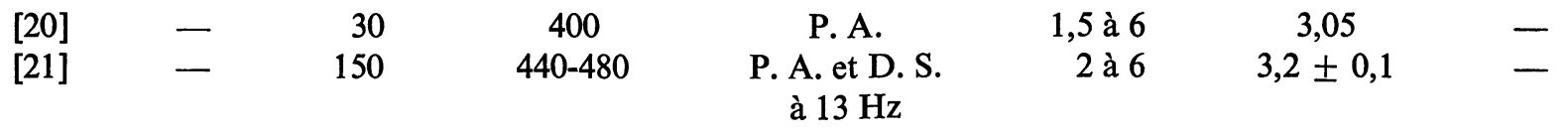

$\Phi_{\mathrm{TE}}:$ hauteur de barrière de l'interface électrode supérieure-oxyde, à champ nul.

$\Phi_{\mathrm{BE}}:$ hauteur de barrière de l'interface électrode de base-oxyde, à champ nul.

$\varepsilon_{\mathrm{r}} \quad:$ constante diélectrique statique de l'alumine.

$e_{\mathrm{TE}}$ : épaisseur de l'électrode supérieure.

$s \quad$ : épaisseur de l'oxyde.

P. A. : mesure du photocourant en régime statique à l'aide d'un picoampèremètre.

D. S. : mesure du photocourant en régime modulé par détection synchrone. 
d'élaboration de l'alumine, sauf pour la pyrolyse pour laquelle la hauteur de barrière $\Phi_{\mathrm{TE}}$ se situe vers $3,2 \mathrm{eV}$.

Certes, les hauteurs de barrière doivent dépendre des états de surface des électrodes d'aluminium ainsi que de la structure cristalline, de la stœchiométrie et de la pureté de l'alumine. Néanmoins, l'influence de ces derniers paramètres ne doit pas être suffisamment importante pour expliquer toute la dispersion des résultats, concernant en particulier $\Phi_{\mathrm{TE}}$.

A ces paramètres liés au mode de préparation de l'alumine, et par conséquent propres à chaque expérimentateur, doivent aussi s'ajouter les difficultés d'interprétation des diagrammes de Fowler liées :

- à la difficulté de localiser l'origine précise des photoélectrons ou des phototrous [19, 22],

- à la non-linéarité de certains graphes $[10,11]$,

- à l'utilisation d'un domaine d'énergie des photons trop restreint et à un nombre de points de mesure insuffisant,

- au caractère quantique du facteur de transmission de la barrière : réflexion et effet tunnel [23, 24],

- à la contribution éventuelle d'une photoconduction extrinsèque de l'alumine comme l'ont fait remarquer certains auteurs $[25,13,26]$.

Ce travail a pour objet d'analyser les photocourants observés dans les structures $\mathrm{Al}-\mathrm{Al}_{2} \mathrm{O}_{3}-\mathrm{Al}$, préparées par bombardement électronique et d'apporter une contribution à l'interprétation des différentes hauteurs de barrière dans la bibliographie.

La technique de préparation de l'alumine par bombardement électronique d'une cible d' $\mathrm{Al}_{2} \mathrm{O}_{3}$ permet :

- de mesurer $\Phi_{\mathrm{BE}}$ indépendamment de l'oxydation de l'électrode d'aluminium de base,

- d'obtenir des structures à isolant épais afin d'étudier l'influence de l'alumine sur le photocourant

- d'utiliser une électrode de base compatible avec des recuits éventuels à haute température de la couche d'alumine.

Cette méthode de préparation de l'oxyde présente les mêmes avantages que la pyrolyse tout en étant plus simple à mettre en œuvre.

2. Méthodes expérimentales. - Les structures $\mathrm{Al}-\mathrm{Al}_{2} \mathrm{O}_{3}-\mathrm{Al}$ sont déposées sur des lames de silice suivant une géométrie en croix. Les électrodes, comme la couche d'alumine, sont évaporées par bombardement électronique dans un groupe à pompage ionique.

Les analyses par rétrodiffusion nucléaire et par diffraction des rayons $\mathbf{X}$ ont montré que les couches d'alumine étaient stœchiométriques et amorphes.

Les épaisseurs de ces couches sont évaluées à partir de la mesure de la capacité de la structure en adoptant pour la constante diélectrique statique de l'alumine : $\varepsilon_{\mathrm{r}}=8,4$, valeur le plus souvent admise (Tableau I). Cette méthode de mesure couramment utilisée est certainement critiquable ; en effet, P. J. Tsang et al. [27] ont montré, que la constante diélectrique statique dépend de l'état plus ou moins amorphe de l'alumine et peut varier de 8 à 12 .

Les échantillons sont étudiés dans un cryostat équipé de fenêtres en silice fondue. Le facteur de transmission $T$ qui intervient dans la définition du rendement de photoémission est déterminé pour une couche d'aluminium de référence préparée en même temps et dans les mêmes conditions que l'électrode supérieure. L'intensité $I_{0}$ de la lumière incidente est mesurée à l'aide d'une thermopile Bi-Ag Eppley.।

La source de rayonnement est constituée d'un monochromateur Bausch et Lomb haute intensité, équipé d'une lampe à arc au Xénon de $150 \mathrm{~W}$ et de deux réseaux utilisés successivement dans les spectres visibles $(0,35 \mu \mathrm{m}$ à $0,80 \mu \mathrm{m})$ et ultra-violet $(0,18 \mu \mathrm{m}$ à $0,40 \mu \mathrm{m})$.

Le monochromateur a été équipé d'un dispositif de balayage automatique de la longueur d'onde dont la vitesse de défilement la plus faible est de $1 \AA / s$. Des filtres sont utilisés pour éliminer les spectres d'ordre supérieur.

Le faisceau lumineux est haché à l'aide d'un modulateur P. A. R. type 191 à une fréquence de l'ordre de $10 \mathrm{~Hz}$.

Le photocourant est mesuré à l'aide d'un détecteur synchrone P. A. R. 124 A équipé du préamplificateur 118.

Les réponses spectrales des structures et les caractéristiques photocourant-tension sont relevées sur un enregistreur $\mathrm{X}-\mathrm{Y}$.

3. Résultats expérimentaux. -3.1 INFLUENCE DU TEMPS ET DE LA FRÉQUENCE DE MODULATION SUR LE PHOTOCOURANT. - La durée des enregistrements du photocourant, liée à la constante de temps choisie pour le détecteur synchrone, pouvant être assez importante (jusqu'à $30 \mathrm{~min}$ ), il est essentiel de s'assurer que les structures n'évoluent pas de façon exagérée au cours de ces enregistrements. A titre d'exemple, dans la figure 1, un enregistrement d'une durée de 15 min est comparé à des mesures point par point effectuées toutes les 5 min à l'aide de filtres interférentiels. Les résultats sont en bon accord, excepté dans la région de $3 \mathrm{eV}$ qui correspond à la fréquence de coupure du filtre placé à la sortie du monochromateur pour éliminer le spectre du second ordre du réseau.

Toutefois des structures polarisées et soumises à un éclairement $I_{0}$ constant pendant des temps beaucoup plus longs ont montré une diminution du photocourant. Cette évolution est illustrée, figure 2 , où sont comparées les variations spectrales du photocourant observées sur une structure particulièrement épaisse $(s=870 \AA)$ juste après sa fabrication (courbe 1) et après $3 \mathrm{~h}$ d'illumination en maintenant une tension de polarisation de $1 \mathrm{~V}$ (courbe 2). On peut noter une diminution du photocourant voisine de $50 \%$. De même, l'amplitude des photocourants diminue lorsque la fréquence de modulation augmente (Fig. 3). Néan- 


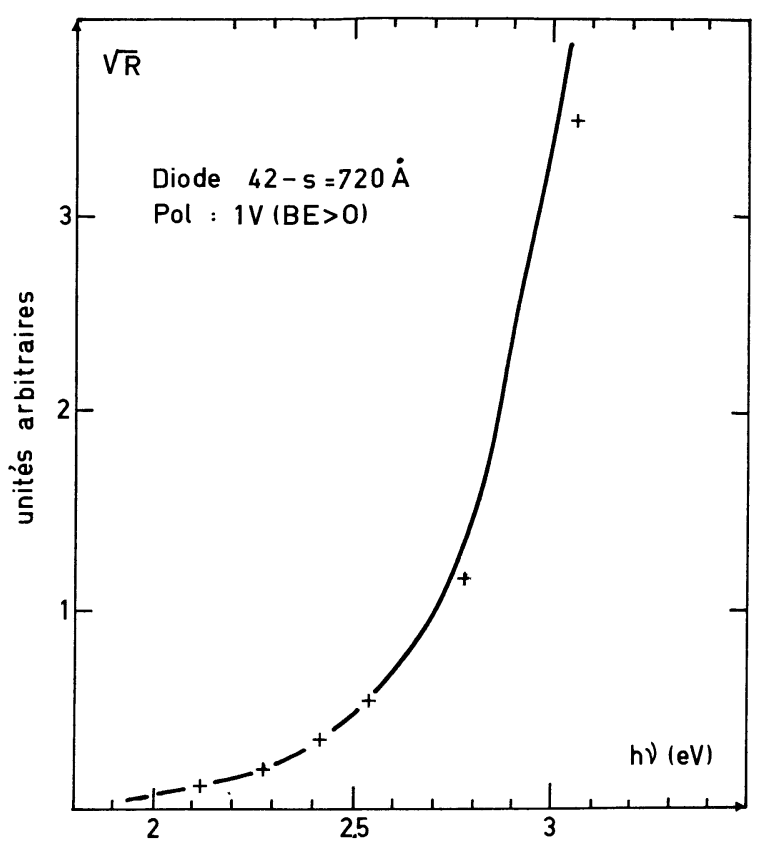

FIG. 1. - Comparaison du rendement de photoémission tiré d'un enregistrement $(5 \AA / \mathrm{s})$ effectué à l'aide d'un monochromateur et de mesures point par point relevées à l'aide de filtres interférentiels.

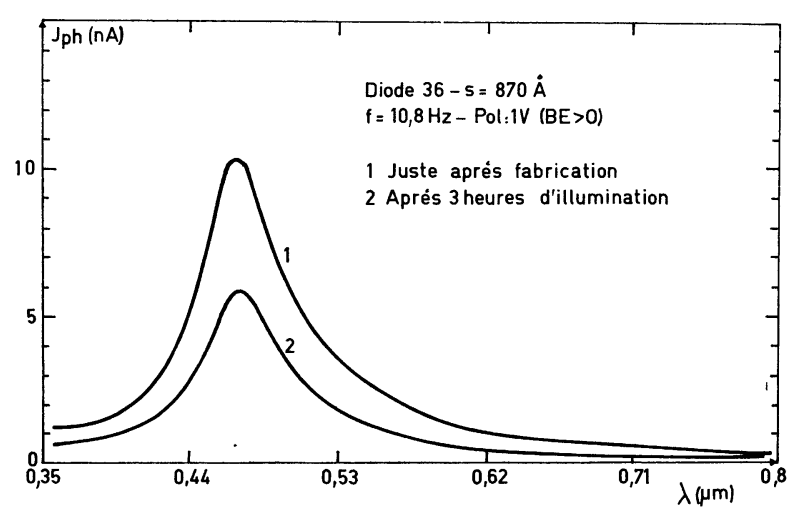

Fig. 2. - Enregistrements effectués à l'aide du réseau visible montrant l'évolution du photocourant au cours du temps.

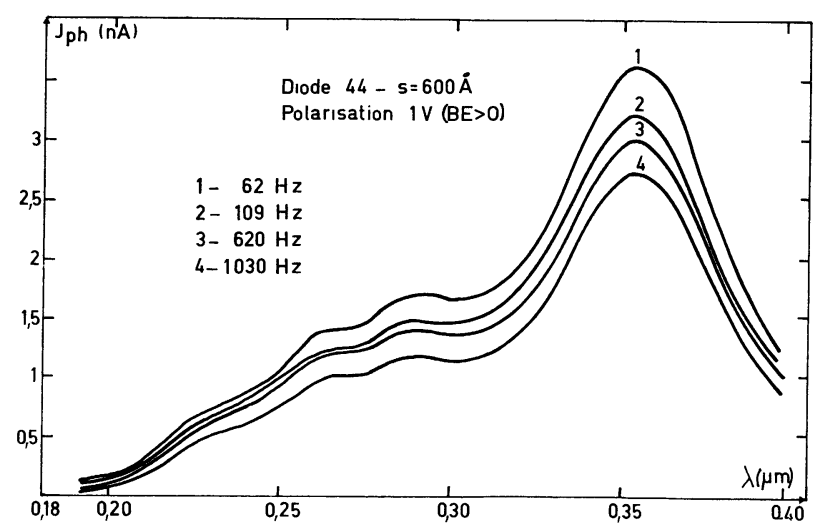

Fig. 3. - Enregistrements effectués à l'aide du réseau ultraviolet montrant la variation des photocourants avec la fréquence de modulation du faisceau lumineux. moins, quel que soit le paramètre, temps ou fréquence de modulation qui conditionne l'évolution des caractéristiques, l'allure générale des réponses spectrales reste inchangée et, par conséquent, le seuil de photoémission n'est pas modifié.

Dans les deux cas, la diminution des photocourants est attribuée en général à l'existence de nombreux pièges dans l'isolant.

3.2 Diagrammes DE Fowler. - Les diagrammes de Fowler relatifs aux structures à isolant mince $(s<300 \AA)$ ne présentent pas de grandes difficultés d'interprétation. Ils sont bien linéaires ainsi que le montrent les courbes (1) et (2) de la figure 4 pour des éner-

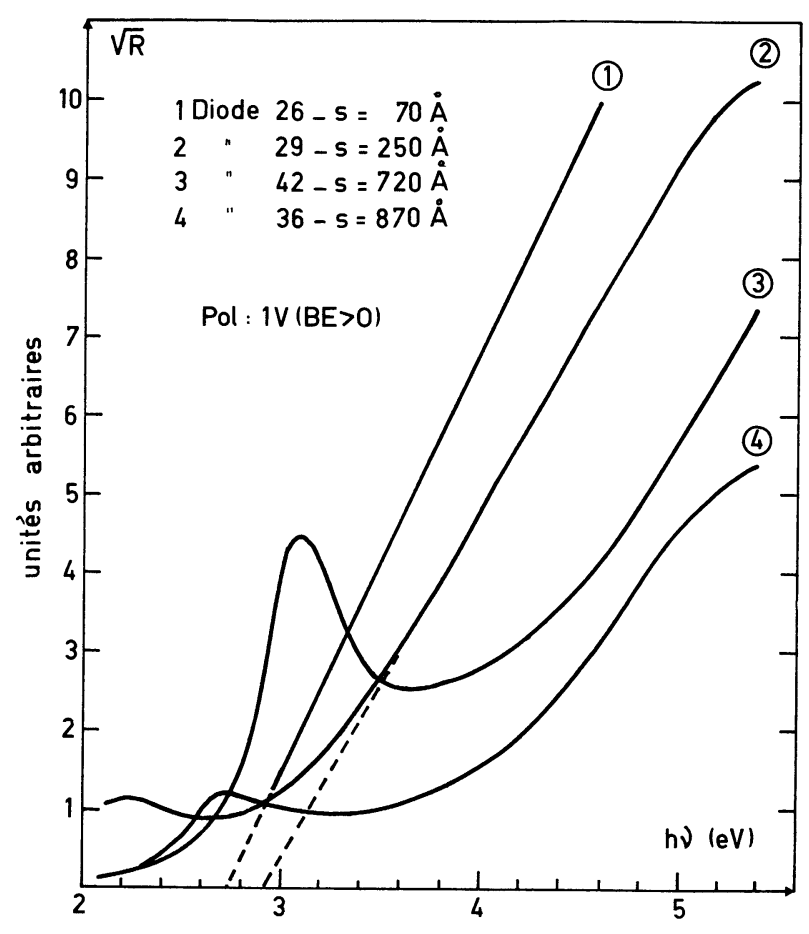

Fig. 4. - Diagrammes de Fowler de quatre échantillons d'épaisseur croissante.

gies supérieures à $3,4 \mathrm{eV}$. L'extrapolation de la partie linéaire permet d'obtenir aisément la hauteur de barrière de l'interface en présence d'une polarisation.

Les diagrammes relatifs aux structures à isolant épais ( $s>300 \AA)$ sont plus complexes comme le montrent les courbes 3 et 4 de la figure 4 . Les parties linéaires sont inexistantes et les maxima de photocourant rendent ces diagrammes inutilisables pour une détermination du seuil de photoémission. Ces diagrammes présentent un photocourant relativement important au voisinage et en dessous du seuil ainsi que des zones à courbure positive pour $h v>3,4 \mathrm{eV}$.

Les courbures positives constatées dans le spectre U. V. $(h v>3,4 \mathrm{eV})$ s'interprètent en faisant intervenir deux processus qui empêchent les photoélectrons d'être collectés. En effet, à la suite de collisions avec les phonons optiques de l'alumine, les photoélectrons perdent de l'énergie $(\Delta E \simeq 0,1 \mathrm{eV})$ et se thermalisent. 
Si cette éventualité se produit dans la région de force image, l'électron aura une très grande probabilité d'être récupéré par l'électrode émissive [10]. Si cette éventualité se produit au-delà de la région de force image, il pourra être capturé par un centre piège situé dans la bande interdite de l'alumine [36].

3.3 CARACTÉRISTIQUES $J_{\mathrm{ph}}=f(V)$. - Dans les figures 5 et 6 sont présentées des caractéristiques

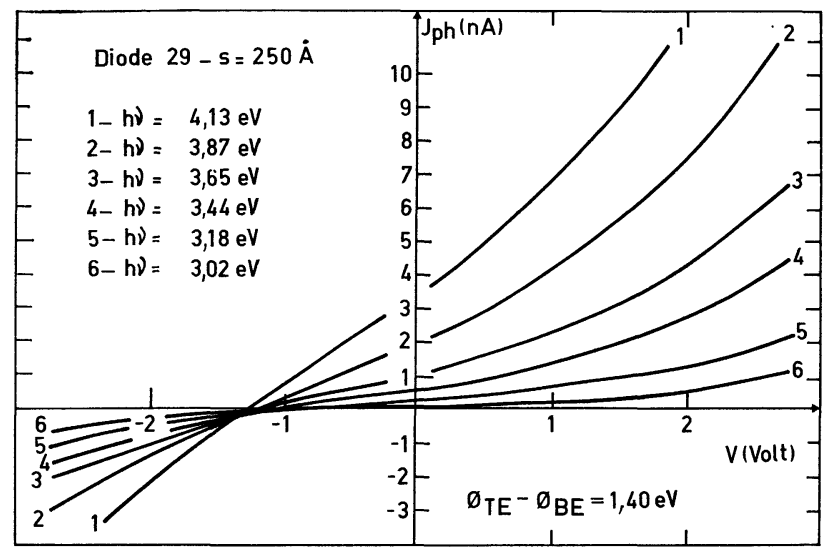

Fig. 5. - Caractéristiques $J_{\mathrm{ph}}=f(V)$ pour un échantillon dont l'électrode de base a été en contact avec l'air. Les tensions positives correspondent à une polarisation positive (polarisation directe) de l'électrode de base. Les courants positifs correspondent à une émission électronique à partir de l'électrode supérieure.

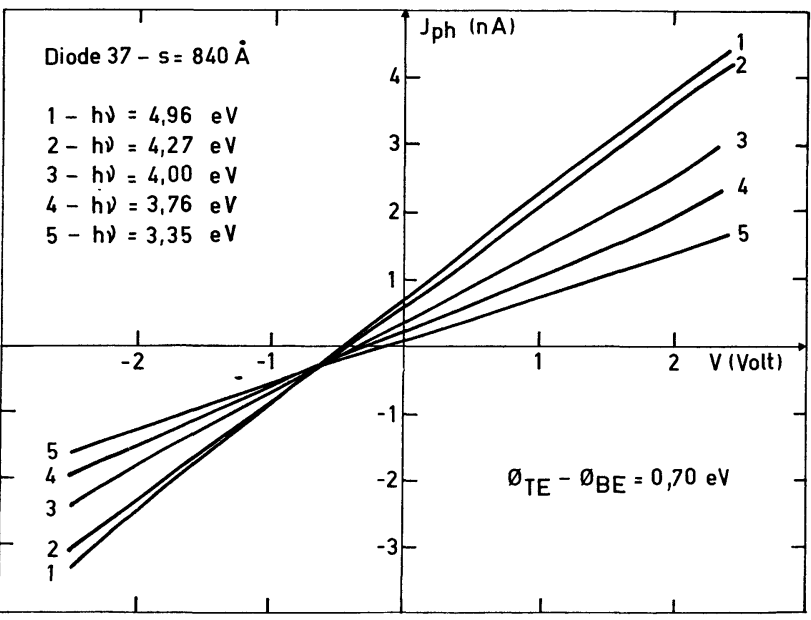

Fig. 6. - Caractéristiques $J_{\mathrm{ph}}=f(V)$ pour une structure préparée intégralement sous vide.

photocourant-tension correspondant à deux échantillons très différents. L'électrode de base de l'échantillon 29 a été exposée à l'air avant évaporation de la couche d'alumine alors que la structure 37 a été réalisée entièrement sous vide. Comme pour toutes les autres structures étudiées, chaque réseau de caractéristiques $J_{\mathrm{ph}}=f(V)$ présente un point commun. L'ordonnée de ce point est voisine de zéro. Son abscisse, toujours négative, caractérise la dissymétrie de la struc- ture. Cette abscisse est liée à la différence des hauteurs de barrière aux deux interfaces :

$$
\left|V_{\mathrm{c}}\right| \simeq \frac{\Phi_{\mathrm{TE}}-\Phi_{\mathrm{BE}}}{e}=\frac{\Delta \Phi}{e} .
$$

Les structures préparées de la même manière que l'échantillon 29 ont présenté comme lui une différence de hauteurs de barrière élevée :

$$
1,2 \mathrm{eV}<\Delta \Phi<1,4 \mathrm{eV} .
$$

Par contre, pour celles préparées dans les mêmes conditions que l'échantillon 37 , cette différence est plus faible :

$$
0,4 \mathrm{eV}<\Delta \Phi<0,7 \mathrm{eV}
$$

En outre, on observe sur les caractéristiques que :

- pour des champs relativement faibles, les caractéristiques sont ohmiques (Fig. 5 et 6),

- pour des champs électriques intermédiaires (Fig. 7) les photocourants suivent une loi empirique de la forme :

$$
J_{\mathrm{ph}}=A \exp \left(a \sqrt{V_{\mathrm{int}}}\right) \quad \text { où } \quad V_{\mathrm{int}}=\frac{\Delta \Phi}{e}+V
$$

$a$ est la pente des parties linéaires des caractéristiques dans les diagrammes $\log J_{\mathrm{ph}}=f\left(V_{\mathrm{int}}^{1 / 2}\right) . V_{\text {int }}$ est la tension effective et $V$ la tension appliquée ;

- pour des champs électriques très élevés, le photocourant est plus grand que celui donné par la loi précédente $\left(\sqrt{V_{\text {int }}}>2 V^{1 / 2}\right.$ Fig. 7$)$.

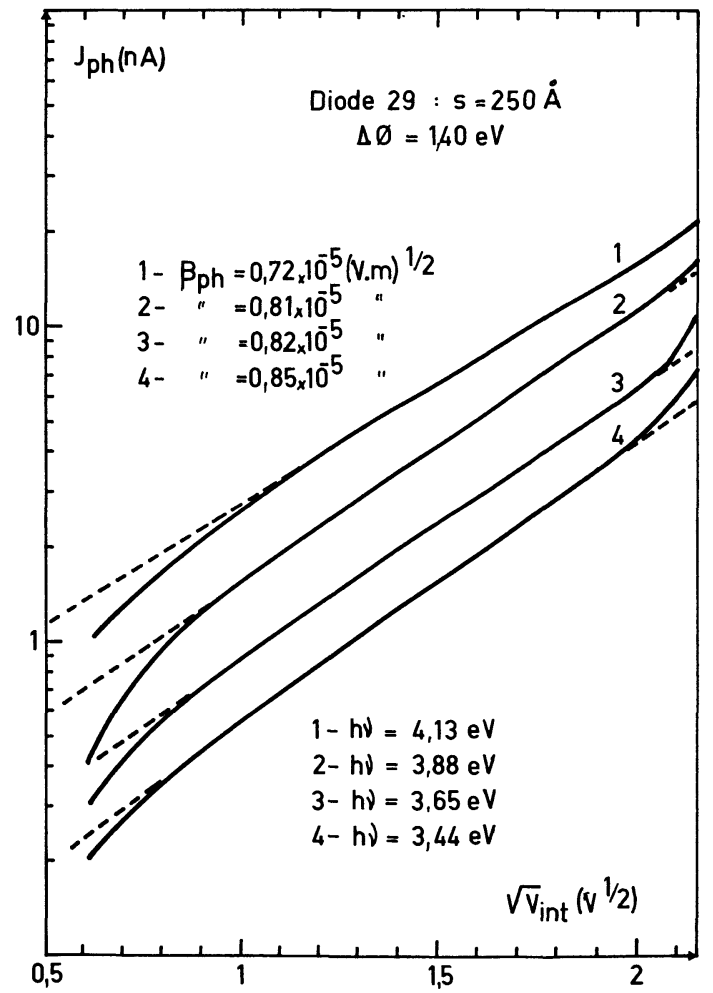

FIG. 7. - Diagrammes du type Schottky ou Poole-Frenkel exploitant les caractéristiques de la figure 5 . 
Comme, par ailleurs, ces trois régions apparaissent également dans l'étude de la variation des courants d'obscurité $J_{\text {obs }}$ avec la tension appliquée [28], il faut alors admettre que les photoélectrons se thermalisent très rapidement ; ils se retrouvent sur les états localisés $\mathrm{du}$ fond de la bande de conduction de l'alumine très près de l'électrode émissive. Les caractéristiques $J_{\mathrm{ph}}=f(V)$ peuvent donc s'interpréter comme les caractéristiques $J_{\text {obs }}=f(V)$.

- Dans la région à faible champ électrique, il semble admis que le processus de conduction est assuré par des sauts (hopping conduction) entre les différents états [29]. Ce mécanisme permet d'expliquer la partie ohmique des caractéristiques $J_{\mathrm{ph}}=f(V)$.

- Le mécanisme qui assure la variation du courant constatée dans la partie intermédiaire des caractéristiques est encore un sujet de polémique. Certains auteurs font intervenir une émission Schottky où la hauteur de barrière de potentiel $\Phi$ des interfaces diminue lorsque le champ électrique augmente. Ce mécanisme conduit à l'expression suivante de la densité de courant :

$$
J_{\mathrm{S}}=A_{\mathrm{S}} \exp \left[-\left(\Phi-\Delta \Phi_{\mathrm{S}}\right) / k T\right] .
$$

L'abaissement Schottky $\Delta \Phi_{\mathrm{S}}$ est défini par :

$$
\Delta \Phi_{\mathrm{S}}=\left(\frac{e^{3} E}{4 \pi \varepsilon_{0} \varepsilon_{\mathrm{r}}}\right)^{1 / 2}=\beta_{\mathrm{S}} E^{1 / 2}
$$

$\varepsilon_{\mathrm{r}}$ est la constante diélectrique optique, $E$ le champ électrique effectif ( $E=V_{\mathrm{int}} / s$, en supposant que le champ électrique est uniforme). Pour l'alumine $\left(\varepsilon_{\mathrm{r}} \simeq 2,6\right)$, le coefficient Schottky est égal à

$$
2,3 \times 10^{-5}(\mathrm{~V} \cdot \mathrm{m})^{1 / 2} .
$$

D'autres auteurs font appel à l'effet Poole-Frenkel qui consiste en une diminution par le champ électrique de la barrière de potentiel coulombienne $\Phi_{\mathrm{PF}}$ associée à chaque piège. On obtient pour un tel mécanisme un courant :

$$
\mathrm{v}_{\mathrm{PF}}=A_{\mathrm{PF}} \exp \left[-\left(\Phi_{\mathrm{PF}}-\Delta \Phi_{\mathrm{PF}}\right) / r k T\right]
$$

où $r$ est un coefficient variant de 1 à 2 [30] suivant la position du niveau de Fermi par rapport à celle des pièges et des donneurs.

L'abaissement Poole-Frenkel $\Delta \Phi_{\mathrm{PF}}$ est défini par :

$$
\Delta \Phi_{\mathrm{PF}}=\beta_{\mathrm{PF}} E^{1 / 2} \text { avec } \beta_{\mathrm{PF}}=2 \beta_{\mathrm{S}} .
$$

Ces deux lois sont pratiquement identiques. Aussi est-il difficile de différencier ces deux mécanismes bien que le second soit un effet de volume théoriquement indépendant de la nature des électrodes métalliques, alors que le premier ne dépend que de l'interface. L'émission Schottky peut néanmoins ne pas dépendre de la nature des électrodes si les états d'interface sont suffisamment nombreux [31].

La loi empirique (2) peut alors s'identifier à une loi du type Schottky ou Poole-Frenkel en définissant un coefficient expérimental $\beta_{\mathrm{ph}}$ déterminé par la relation :

$$
\beta_{\mathrm{ph}}=a k T \sqrt{s} .
$$

Expérimentalement (Fig. 7), on constate que les coefficients $\beta_{\mathrm{ph}}$ sont inférieurs à $\beta_{\mathrm{S}}$ et à fortiori à $\beta_{\mathrm{PF}}$ et qu'ils varient suivant l'énergie des photons incidents. Ces observations rejoignent celles de A. K. Jonscher et A. A. Ansari [32] qui les expliquent en attribuant aux photoélectrons une température électronique supérieure à la température thermodynamique (électrons chauds).

- Lorsque le champ électrique augmente, les écarts à la loi empirique (2) pourraient se justifier par un phénomène d'effet tunnel à travers la barrière qui s'amincit ou à l'aide d'un modèle basé sur le concept d'électron chaud [28].

4. Exploitation des résultats. - Tous les échantillons minces, préparés par bombardement électronique, ont présenté sensiblement la même hauteur de barrière à champ nul $\Phi_{\mathrm{TE}}$ associée à l'interface électrode supérieure-alumine. Les différents seuils de photoémission corrigés de l'abaissement Schottky (4) résultant du champ électrique appliqué, et du champ électrique interne déterminé à partir de l'abscisse du point commun des caractéristiques $J_{\mathrm{ph}}=f(V)$, conduisent à

$$
\Phi_{\mathrm{TE}}=3,2 \pm 0,2 \mathrm{eV} .
$$

Nous avons retrouvé cette valeur (Figs. 8 et 9) pour des structures dont la couche d'alumine a été obtenue par électrolyse de l'électrode de base [33]. La valeur de la hauteur de barrière : $3 \mathrm{eV}$ de l'interface électrode supérieure-alumine à polarisation nulle (encart de la Fig. 8) doit être corrigée également de l'effet Schottky dû au champ interne. Ce dernier résulte de la différence des hauteurs de barrière $\Delta \Phi=1,3 \mathrm{eV}$ déterminée à partir du réseau de diagrammes de Fowler relevés avec une polarisation négative (Fig. 9).

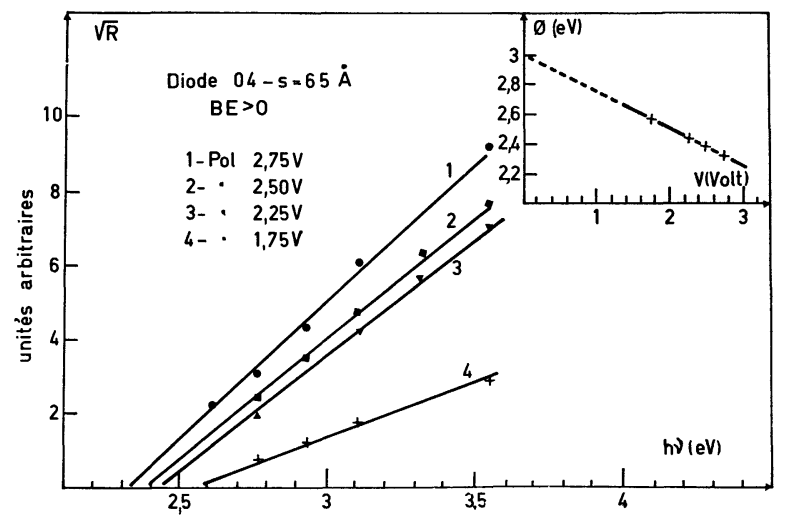

Fig. 8. - Diagrammes de Fowler (relevés avec une polarisation directe) d'une structure dont l'isolant est obtenu par électrolyse, permettant de déterminer la hauteur de barrière à polarisation nulle de l'interface électrode supérieure-alumine $\Phi_{\mathrm{TEE}}$. 


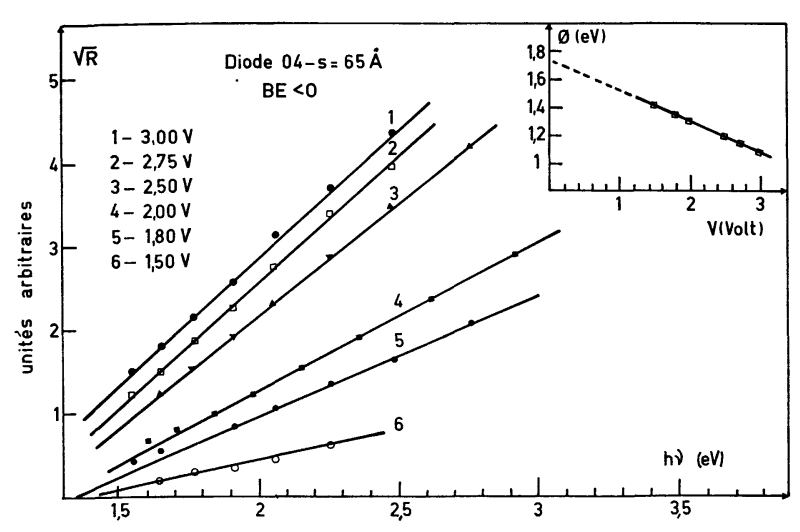

FIG. 9. - Diagrammes de Fowler (relevés avec une polarisation inverse) $(\mathrm{BE}<0)$ de la structure 04, permettant de déterminer la hauteur de barrière à polarisation nulle de l'interface électrode de base-alumine $\Phi_{\mathrm{BE}}$.

De plus, comme cette valeur de $\Phi_{\mathrm{TE}}$ est la même que celle obtenue pour l'interface aluminium-alumine pyrolytique (Tableau I), il apparaît que $\Phi_{\mathrm{TE}}$ dépend assez peu du mode de fabrication de l'alumine.

Par contre $\Phi_{\mathrm{BE}}$, la hauteur de barrière associée à l'interface électrode d'aluminium de base-alumine, est une grandeur qui dépend énormément des conditions de préparation. Les caractéristiques $J_{\mathrm{ph}}=f(V)$ ont montré que la différence $\Delta \Phi=\Phi_{\mathrm{TE}}-\Phi_{\mathrm{BE}}$ pouvait varier entre $0,4 \mathrm{eV}$ et $1,4 \mathrm{eV}$ suivant le degré d'oxydation de l'électrode d'aluminium de base. Les valeurs les plus faibles de $\Phi_{\mathrm{BE}}$ ont été mesurées sur des structures pour lesquelles l'électrode de base a été en contact avec l'air humide à la pression atmosphérique. Il est alors naturel que la valeur $1,8 \mathrm{eV}$ trouvée dans ces conditions soit en bon accord avec la moyenne des valeurs rencontrées dans la bibliographie (Tableau I) pour des structures dont l'alumine est obtenue par oxydation de l'électrode de base. Ce résultat est à comparer à celui de Huber et al. [34] qui ont mesuré le travail de sortie de l'aluminium oxydé en présence de vapeur d'eau; ce dernier est inférieur de $1,2 \mathrm{eV}$ à celui d'une surface propre d'aluminium.

Donc, si on peut admettre que le mode et les conditions de préparation de la structure peuvent être rendus responsables des valeurs très différentes rencontrées dans la bibliographie pour $\Phi_{\mathrm{BE}}$, il n'en est pas de même pour $\Phi_{\mathrm{TE}}$.

Pour expliquer la dispersion des valeurs de $\Phi_{\mathrm{TE}}$ consignées dans le tableau $\mathrm{I}$, on peut faire appel à l'hypothèse de M. Kleefstra et al. [13] et E. Klein et al. [26] qui admettent que les photocourants mesurés résultent de la superposition de phénomènes de photoconduction extrinsèque dans le volume de l'alumine et de photoémission à travers une interface.

En effet, les diagrammes de Fowler, relevés en particulier sur des structures à forte épaisseur d'oxyde $(s>200 \AA)$ pour lesquelles la photoconduction doit être marquée, font apparaitre (Fig. 4) des photocourants importants pour des énergies de photons voisines ou inférieures au seuil. Cette observation peut s'appliquer aux diagrammes de Fowler publiés par d'autres auteurs $[11,13,26]$ sur de l'alumine amorphe. Par contre, N. Szydlo et R. Poirier [20] ont obtenu des diagrammes de Fowler bien linéaires même près du seuil, mais pour des structures dont l'alumine obtenue par pyrolyse est polycristalline. Cette différence peut s'expliquer en admettant par exemple la présence de niveaux d'énergie dans la bande interdite des couches d'alumine amorphe, niveaux qui donneraient lieu à des transitions électroniques.

Cette hypothèse est renforcée par l'allure des spectres d'absorption optique relevés sur des couches d'alumine très épaisses $(1 \mu \mathrm{m}$ à $5 \mu \mathrm{m})$ préparées dans les mêmes conditions et à partir de la même cible que celles étudiées par photoémission (Fig. 10). Ces spectres sont

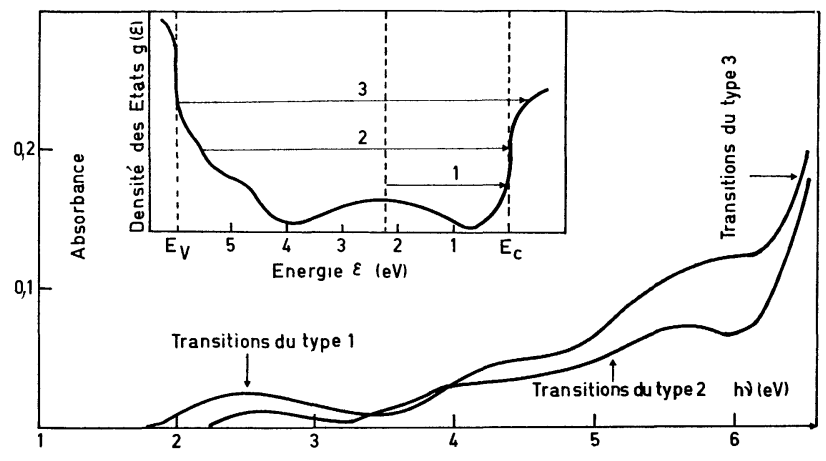

Fig. 10. - Variations spectrales de l'absorbance de deux couches d'alumine. La figure dans l'encart donne une interprétation des bandes d'absorption.

caractérisés par une bande de faible absorbance ( $2 \%)$ au voisinage de $2,5 \mathrm{eV}$, suivie d'un front graduel plus ou moins monotone entre $3,5 \mathrm{eV}$ et $6 \mathrm{eV}$ et enfin par l'amorce d'une augmentation brusque de l'absorbance à partir de $6,2 \mathrm{eV}$. Les positions des divers maxima varient suivant la couche d'alumine étudiée.

Ces spectres d'absorption peuvent s'interpréter en faisant intervenir 3 types différents de transitions (encart de la Fig. 10) Les transitions du type 1 concernent les états localisés situés vers le milieu de la bande interdite, celles du type 2 les états localisés qui constituent la queue de la bande de valence de l'alumine amorphe et celles du type 3 sont relatives aux états mobiles de la bande de valence. En adoptant ce modèle, on peut attribuer aux couches d'alumine amorphe réalisées par bombardement électronique une largeur de bande interdite de 6,2 eV. Comme il est admis [19] que la largeur de bande interdite de l'alumine varie suivant le mode de fabrication : 5,2 eV pour de l'alumine plasma, $6 \mathrm{eV}$ à $7 \mathrm{eV}$ pour de l'alumine électrolytique et $8 \mathrm{eV}$ à $9 \mathrm{eV}$ pour la phase cristalline, la valeur de $6,2 \mathrm{eV}$ est acceptable pour de l'alumine amorphe.

La densité des états localisés dans la bande interdite dépend de façon critique des conditions de préparation 
(température du substrat et recuit), comme l'ont montré Le Comber et al. [35] pour des films amorphes de silicium.

Effectivement, le recuit a une très grande influence sur les bandes d'absorption de l'alumine, comme le montre la figure 11. C'est ainsi qu'un recuit dans l'air

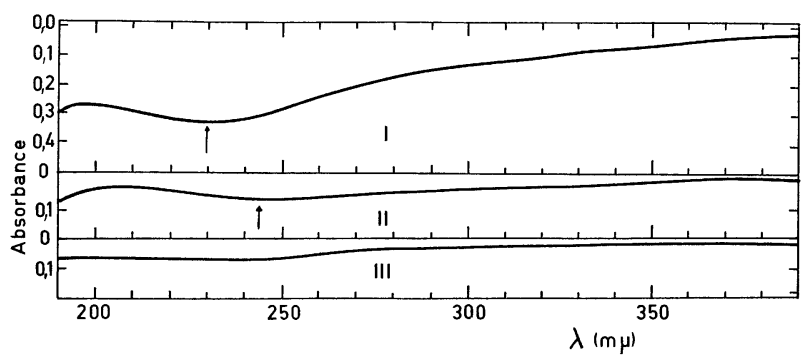

FIG. 11. - Modification de l'absorbance d'une couche d'alumine au cours de recuits successifs. I) Couche $\mathrm{Al}_{2} \mathrm{O}_{3}$ avant recuit ; II) Même couche après recuit de $12 \mathrm{~h}$ à $400^{\circ} \mathrm{C}$ à l'air ; III) Même couche après un second recuit de $12 \mathrm{~h}$ à $800^{\circ} \mathrm{C}$ à l'air.

à $400^{\circ} \mathrm{C}$ pendant $12 \mathrm{~h}$ d'une couche d'alumine diminue son absorbance et déplace la bande d'absorption (courbe II). Un recuit ultérieur de la même lame à $800^{\circ} \mathrm{C}$ pendant $12 \mathrm{~h}$ a pratiquement fait disparaître la bande d'absorption (courbe III) ainsi que l'amorce du front d'absorption vers $0,2 \mu \mathrm{m}(6,2 \mathrm{eV})$. Cette évolu- tion de l'absorption traduit une modification de structure de l'alumine dans le sens d'une amélioration de l'ordre (augmentation de la largeur de bande interdite) et d'une saturation des liaisons pendantes. Cependant, nous n'avons pas observé de changements dans les dingrammes de rayons $X$ effectués après chaque opération.

5. Conclusions. - Ce travail confirme que la hauteur de barrière de l'interface $\mathrm{Al}-\mathrm{Al}_{2} \mathrm{O}_{3}$ se situe vers $3,2 \mathrm{eV}$. Cette hauteur semble dépendre assez peu de la méthode d'élaboration de la couche d'alumine.

Lorsque l'électrode d'aluminium est oxydée, en présence notamment de vapeur d'eau, la hauteur de barrière diminue jusque vers $1,8 \mathrm{eV}$.

Remerciements. - Les auteurs remercient MM. les Pr. H. Carchano, R. Garron et S. Martinuzzi pour les fructueuses discussions sur les phénomènes de conduction dans les amorphes, de photoémission et de piégeage. Ils expriment leur reconnaissance à M. B. Pichaud du Laboratoire de Cristallographie de l'Université d'Aix-Marseille III pour l'analyse par diffraction des rayons $\mathrm{X}$ et $\mathrm{MM}$. J. C. Oberlin, J. P. Stoquert et A. Genoux-Lubin du Département de Physique Nucléaire du C. S. T. N. à Alger pour les analyses par rétrodiffusion nucléaire.

\section{Bibliographie}

[1] Sze, S. M., Physics of Semiconductors Devices (Wiley interscience) 1969.

[2] Goodman, A. M., J. Electrochem. Soc. 115 (1968) 276C.

[3] Williams, R., Semiconductors and semimetals by R. K. Willardson and A. C. Beer (Ac. Press N. Y. London) 1970 vol. 6, p. 97.

[4] Gaudart, L., Thèse de doctorat, Marseille 10 décembre 1975.

[5] BEDos, R., 6th International IMEKO Symposium on photon detectors Sirfök Humgary, 1974.

[6] Gundlach, K. H. and KadleC, J., J. Appl. Phys. 46 (1975) 5286.

[7] ShePard, K. W., J. Appl. Phys. 36 (1965) 796.

[8] Ludwig, W. and Korneffel, B., Phys. Stat. Sol. 24 (1967) K 137.

[9] SChuermeyer, F. L., J. Appl. Phys. 37 (1966) 1998.

[10] SChuermeyer, F. L., Young, C. R. and Blasingame, J. M., J. Appl. Phys. 39 (1968) 1791.

[11] Nelson, O. L. and Anderson, D. F., J. Appl. Phys. 37 (1966) 77.

[12] Musatov, A. L., Sov. Phys. Sol. Stat. 9 (1968) 2580.

[13] Kleefstra, M., Vanzanten, A. T. and Ceriolo, P., Thin Solid Films 30 (1975) 225.

[14] Braunstein, A., Braunstein, M., Picus, G. S. and Mead C. A., Phys. Rev. Lett. 14 (1965) 219.

[15] Braunstein, A. I., Braunstein, M. and Picus, G. S., Phys. Rev. Lett 15 (1965) 956.

[16] Gundlach, K. H. and Holz, J., Surf. Sci. 27 (1971) 125.

[17] Korneffel, B. and Ludwig, W., Phys. Stat. Sol. (a) 8 (1971) 149.

[18] RouzeYre, M., Thèse de doctorat Montpellier 5 Nov. 1966.
[19] Goodman, A. M., J. Appl. Phys. 41 (1970) 2176.

[20] Szydlo, N. and Poirier, R., J. Appl. Phys. 42 (1971) 4880.

[21] Dimaria, D. J., J. Appl. Phys. 45 (1974) 5454.

[22] Gundlach, K. H. and Kadlec, J., Thin Solid Films 28 (1975) 107.

[23] Kadlec, J. and Gundlach, K. H., Phys. Stat. Sol. (a) 37 (1976) 11.

[24] Burshtein, Z. and Levinson, J., Phys. Rev. B 128 (1975) 3453.

[25] Musatov, A. L. and Tsvetaev, S. K., Sov. Phys. Solid State 12 (1970) 242.

[26] Klein, E., Vodenicharov, C., Ikonopisov, S., Trifonova V. and Christov, S. G., Phys. Stat. Sol. 32 (1975) K 131.

[27] Tsang, P. J., Anderson, R. M. and Cvikevich, S., $J$. Electrochem. Soc. 123 (1976) 57.

[28] Antula, J. J. Appl. Phys. 43 (1972) 4663.

[29] Mead, C. A., Phys. Rev. 128 (1962) 2088.

[30] Simmons, J. G., Phys. Rev. 155 (1967) 657.

[31] Hartman, T. E., Blair, J. C. and Bauer, R., J. Appl. Phys. 37 (1966) 2468.

[32] Jonscher, A. K. and Ansari, A. A., Phil. Mag. 23 (1971) 205.

[33] Bernard, J., Haug, R., Mentalecheta, Y. and TreGOUET, Y., Phys. Stat. Sol. (a) 6 (1971) K 127.

[34] Huber, E. E., Jr. and KIRK, C. T., Surf. Sci. 5 (1966) 447.

[35] Lecomber, P. G., MAdAN, A. and SpeAR, W. E., Electronic and structural properties of amorphous semiconductors (Academic Press) 1973 ch. 10, p. 373.

[36] Powell, R. J. et Berglund, C. N., J. Appl. Phys. 42 (1971) 4390. 